\title{
Reply to the correspondence letter by J. Finsterer and S. Zarrouk-Mahjoub "Noncompaction in mitochondrial trifunctional protein deficiency due to a HADHB mutation"
}

\author{
Tiina Ojala ${ }^{1}$
}

Received: 14 July 2015 / Accepted: 16 July 2015 / Published online: 31 July 2015

(C) Springer-Verlag Berlin Heidelberg 2015

We want to thank the authors for their carefully provided comments on our manuscript "Fetal left ventricular non compaction cardiomyopathy and fatal outcome due to complete deficiency of mitochondrial trifunctional protein."

The authors asked why left ventricular hypertrabeculation/ noncompaction was not diagnosed already prenatally during fetal echocardiography? As mentioned in the manuscript, fetal cardiac echocardiography showed significant biventricular cardiac hypertrophy already in the first fetal echocardiography at 22 gestational weeks. However, left ventricular noncompaction was overlooked and was not diagnosed before the patient was born. As pointed out by the authors, left ventricular noncompaction should be suspected if ventricular walls are thickened. We found the left ventricular noncompaction in postnatal echocardiography as shown in Figure 2 in the manuscript. In the postmortem examinations, cardiac findings were not in focus and we are not aware whether cardiomyopathy findings could even be conclusively studied by postmortem MRI.

Both parents were heterozygous carriers of the mutation (HADHB gene, c.1109+243_1438-703del). We are not aware of any report in the literature that heterozygous carriers would have any abnormalities. Both the parents and the younger sister are without symptoms. The echocardiography with normal findings was done to the sister.

Three different phenotypes have been described: the lethal phenotype, the infant-onset hepatic phenotype, and the lateronset neuromyopathic phenotype. The lethal phenotype is characterized by severe cardiomyopathy, lactic acidosis, hypoketotic hypoglycemia, and neonatal death. The phenotype of our patient was the most severe form of TFP deficiency.

We appreciate that we have been given the opportunity for these clarifications.

Tiina Ojala, MD, PhD

Communicated by Jaan Toelen

This is a reply to a correspondence which can be found at http://dx.doi. org/10.1007/s00431-015-2598-1.

Tiina Ojala

tiina.h.ojala@hus.fi

1 Children's Hospital, University Hospital of Helsinki,

Helsinki, Finland 